\title{
What Drives an Aliquot Sequence?
}

\author{
By Richard K. Guy* and J. L. Selfridge \\ To D. H. Lenmer, on his 70 th birthday, in gratitude for much inspiration, \\ encouragement and computation
}

\begin{abstract}
The concept of the "driver" of an aliquot sequence is discussed. It is shown that no driver can be expected to persist indefinitely. A definition of driver is given which leads to just 5 drivers apart from the even perfect numbers.
\end{abstract}

If we examine the sequence $30,42,54,66,78,90, \ldots$ we notice that each term is the sum of the aliquot parts (divisors other than the number itself) of its predecessor. Various authors have been struck by the peculiar charm and regularity displayed by these aliquot sequences. It is easy to show that every such sequence starting with a number less than 138 either contains 1 and terminates, or contains a perfect number and repeats.

Catalan's conjecture [1] was restated by Dickson [3] to the effect that every aliquot sequence will either terminate or become periodic; e.g., the amicable pair 220 , 284 has period 2. Later Poulet [8] found that 12496 starts a sequence with period 5 and that 14316 has period 28 . He and Lehmer struggled with the sequence starting with 138 .

More formally, let $s(n)=\sigma(n)-n$, where $\sigma(n)$ is the sum of the divisors of $n$, and let $n: 0=n, n: k+1=s(n: k)$. Lehmer showed that the 138 sequence has a maximum

$$
179931895322=138: 117=2 \cdot 61 \cdot 929 \cdot 1587569
$$

and that $138: 177=1$.

The next difficulty arose with the 276 sequence. Each term from $2716=$ $276: 8=2^{2} 7 \cdot 97$ is divisible by $2^{2} 7$, and since any multiple of the perfect number 28 is abundant, the terms increase monotonically. Notice that in our little sequence starting with 30 the terms have the same property with respect to 6. Paxson [7] computed ( $p$ denotes a prime cofactor)

$$
5641400009252=276: 67=2^{2} 7 \cdot p
$$

and Henri Cohen [2] extended the calculation to

Received June 3, 1974.

AMS (MOS) subject classifications (1970). Primary 10A20; Secondary 10-04, 10L10.

Key words and phrases. Aliquot sequence, guide, driver, perfect number, amicable pair, Poulet cycle, Catalan-Dickson conjecture.

* Research of the first author supported by grant A-4011 of the National Research Council of Canada. 


$$
2133148752623068133100=276: 118=2^{2} 3 \cdot 5^{2} 7 \cdot p .
$$

Stimulated by this, Lehmer persevered until he reached the term $276: 169=$ $2^{2} 7^{2} p$, where $p$ is a prime congruent to $1, \bmod 4$, so that

$7421365124006306789124764410=276: 170=2 \cdot 5 \cdot 7 \cdot 13 \cdot 829 \cdot 848557 \cdot p$ suddenly lost the "driver" 28 . The succeeding terms, all being congruent to \pm 2 , mod 12, decrease steadily from $276: 172$ to the term $276: 226=2 p$, where again $p$ is a prime congruent to $1, \bmod 4$, and

$$
351121244430380=276: 227=2^{2} 5 \cdot 131 \cdot 48539 \cdot 2760991 .
$$

Lehmer has computed a further 200 terms which show an erratic upward tendency. The extent of our present knowledge [5], [6] is

$$
107100047962427456048833497403019424=276: 433=2^{5} 3 \cdot 199 \cdot c
$$

where $c$ is a 31-digit composite number with no small factors. Lehmer also verified that, apart from $396=s(276)=s(306)$, all sequences starting with numbers less than 552 are bounded.

On the other hand, we have found [4], [5], [6] that of the sequences starting with numbers less than $10^{4}$, there are 751 which contain a term exceeding $10^{24}$; and we have conjectured that an infinite number of aliquot sequences are unbounded. Our aim here is to outline some of the characteristics of the "driver" phenomenon which support this view. Good examples of driver dominated sequences are

$$
\begin{aligned}
628628 & =552: 26=2^{2} 7 \cdot 11 \cdot 13 \cdot 157 \\
35149477396986268016618686344127020 & =552: 181=2^{2} 3^{2} 5 \cdot 7^{2} c, \\
3985297814226 & =564: 83=2 \cdot 3 \cdot 211 \cdot 3147944561 \\
252663526 & =564: 265=2 \cdot 3^{2} 23 \cdot 89 \cdot c, \\
11400 & =5250: 3=2^{3} 3 \cdot 5^{2} 19 \\
2422499075303417661059 & =5250: 72=2^{3} 3 \cdot 5 \cdot c, \\
8154 & =8154: 0=2 \cdot 3^{3} 151 \\
4553462993488753886439512520 & =515: 201=2 \cdot 3^{6} 5 \cdot 43 \cdot c, \\
1503680 & =8904: 13=2^{6} 5 \cdot 37 \cdot 127 \\
4615096670497664245830510 & =8154,166=2^{6} 89 \cdot 127 \cdot c, \\
4200141507007701992846912 & =8904: 144=9852: 11=2^{4} 31 \cdot 89 \\
5149877193773848066488144 & =9852: 146=2^{4} 3 \cdot 11 \cdot 31 \cdot c .
\end{aligned}
$$

Despite the tenacity of these drivers, none is expected to live for ever.

We notice that any prime divisor $p$ of $n$ will appear in $s(n)$ just if $p$ divides $\sigma(n)$ and will appear to the same power in $s(n)$ if a higher power divides $\sigma(n)$. If $p$ divides both $n$ and $\sigma(n)$ to the same power, $p$ will divide $s(n)$ to at least that power and to a higher power with probability $1 /(p-1)$, i.e., 
always when $p=2$. In fact, the prime 2 will continue to be present unless $n$ is a square or twice a square, and continue to be absent unless $n$ is a square. This fact, more than any other, seems to dominate the discussion of the behavior of aliquot sequences.

A very rough argument in favor of our conjecture goes like this: on the average the value of $\sigma(n)-n$ is greater than $n$ if $n$ is even, and less than $n$ if $n$ is odd. If in the long run other effects are small compared to the persistence of parity, one would expect that most large even sequences are unbounded and that most odd sequences are bounded.

More precisely, since the average order, $\alpha$, of $s(n) / n$, taken over even values of $n$, is greater than one so long as the terms remain even, we expect $n: r$ to be $n \alpha^{r}$. The probability that this is a square, or twice a square, is $c_{1} / \sqrt{n \alpha^{r}}$, so that the probability that any future term is odd is $c_{2} / \sqrt{n}$, which tends to 0 as $n \rightarrow \infty$.

One might ask if a sequence could be shown to be unbounded by displaying a driver which persisted indefinitely. This would only occur if certain prime factors of $n$ would always continue to appear to the same or higher powers in $s(n)$. The prime 2 in fact should keep the same power throughout, since the nature of the driver changes radically when the power of 2 changes. If each of a set of primes divides $\sigma(n)$ to a higher power than it divides $n$, then we would have achieved the goal. We prove that this cannot happen.

THEOREM 1. For any divisor $v, v>1$, of $n$, there is some prime divisor of $v$ which does not divide $\sigma(v) / v$.

Proof. Let $v=2^{a} p_{1}^{a_{1}} \cdots p_{r}^{a_{r}}$ with $a_{1}, \cdots, a_{r}>0$, so that

$$
\sigma(v)=\left(2^{a+1}-1\right) \frac{p_{1}^{a_{1}+1}-1}{p_{1}-1} \cdots \frac{p_{r}^{a_{r}+1}-1}{p_{r}-1} .
$$

If $p_{1} \cdots p_{r} \mid \sigma(v) / v$, then

$$
p_{1} \cdots p_{r} \leqslant \frac{\sigma(v)}{v}<2 \frac{p_{1}}{p_{1}-1} \cdots \frac{p_{r}}{p_{r}-1},
$$

i.e. $\left(p_{1}-1\right) \cdots\left(p_{r}-1\right)<2$ and $v$ has no odd prime divisors. Moreover, since $\sigma\left(2^{a}\right)$ is odd, $2 \nmid v$.

Note that we can prove this without the requirement that the power of 2 be higher in $\sigma(v)$ than in $v$.

A precise definition of driver is desired at this point, but we would like our definition to avoid fragile structures, such as

$$
2^{7} 3^{6} 5 \cdot 17 \cdot 23 \cdot 137 \cdot 547 \cdot 1093
$$

which crumples when the power of 3 changes. In addition to the even perfect numbers one would normally include in a list of drivers, any products of prime powers which have a reasonable expectation of persistence, such as $2^{3} 3 \cdot 5,2^{5} 3 \cdot 7$ and $2^{9} 3 \cdot 11 \cdot 31$. 
Each of these divides the sum of its divisors and hence divides the sum of its aliquot parts.

For example, if $n=2^{9} 3 \cdot 11 \cdot 31 \cdot m$, where $\left(m, 2^{11}-2\right)=1$, then $\sigma(n)=$ $2^{9} 3^{2} 11 \cdot 31 \cdot \sigma(m)$, where $\sigma(m)$ is even when $m$ is not a square. Then $s(n)=$ $2^{9} 3 \cdot 11 \cdot 31 \cdot m^{\prime}$ where $\left(m^{\prime}, 6\right)=1$ and the chance of $m^{\prime}$ being divisible by 11 or 31 is small, and of its being a square is negligible. For similar reasons $2^{3} 3 \cdot 5$ and $2^{5} 3 \cdot 7$ are persistent.

A further remarkable driver is the number 2. When $n=2 m$ and $(m, 6)=1$, $\sigma(n)=3 \sigma(m)$ and $s(n)=2 m^{\prime}$, where $\left(m^{\prime}, 6\right)=1$ provided that $4 \mid \sigma(m)$. Neglecting squares, $4 \mid \sigma(m)$ unless $m$ is a prime congruent to 1 , mod 4 . Similar considerations hold for $2^{3} 3$.

If $n=2^{3} 3 \cdot 5 \cdot m$, we find it convenient to regard $2^{3} 3 \cdot 5$ as the driver even when $(m, 15) \neq 1$ (and similarly for other drivers) so that the only crucial exponent is that of 2. That is, we draw the line to exclude some possibilities which tempt us on account of their stability, but which rely for this on considerations secondary to the factorization of $\sigma\left(2^{a}\right)=2^{a+1}-1$. Some further examples which are thus excluded are $2^{3} 3^{2} 5 \cdot 13,2^{5} 3^{2} 7 \cdot 13,2^{5} 3^{3} 5$, and $2^{5} 3^{3} 5 \cdot 7$.

Define a guide to be $2^{a}$, together with a subset of the prime factors of $\sigma\left(2^{a}\right)$ A driver is defined as a number $2^{a} v$ with $a>0, v$ odd, $v \mid \sigma\left(2^{a}\right)$ and $2^{a-1} \mid \sigma(v)$. This last requirement is included so that the power of the prime 2 will tend to persist at least as well as it does for the driver 2 itself, for which the condition is trivially satisfied.

THEOREM 2. The only drivers are $2,2^{3} 3,2^{3} 3 \cdot 5,2^{5} 3 \cdot 7,2^{9} 3 \cdot 11 \cdot 31$, and the even perfect numbers.

Proof. Let $2^{a} v$ be a driver, so that $v\left|2^{a+1}-1,2^{a-1}\right| \sigma(v)$. If $2^{a+1}-1=v$ is a Mersenne prime, the driver is an even perfect number. If $v=1,2^{a-1} \mid \sigma(v)=1$, $a=1$ and we have the "downdriver" 2 . Henceforth we assume that $v>1$, and that $\sigma\left(2^{a}\right)=2^{a+1}-1=p_{1}^{a_{1}} \cdots p_{r}^{a_{r}}$ is composite, so that $v=p_{1}^{b_{1}} \cdots p_{r}^{b_{r}}, 0 \leqslant b_{i} \leqslant a_{i}$, $1 \leqslant i \leqslant r$ and not all the $b_{i}$ are zero.

Define the deficiency of the factor $p_{i}^{b_{i}}$ of $v$ to be $2^{d_{i}} / p^{a_{i}}$, where $2^{d_{i}}$ is the highest power of 2 in any $\sigma\left(p_{i}^{j}\right), 0 \leqslant j \leqslant b_{i}$. The product of the deficiencies of the factors of $v$ is greater than $1 / 4$, since otherwise

$$
2^{a+1}>2^{a+1}-1=\prod_{i=1}^{r} p_{i}^{a_{i}} \geqslant 4 \prod_{i=1}^{r} 2^{d},
$$

so that $2^{a-1}>\Pi_{i=1}^{r} 2^{d_{i}}$ and $2^{a-1} \nmid \Pi_{i=1}^{r} \sigma\left(p_{i}^{b_{i}}\right)=\sigma(v)$.

The product of the deficiencies of the Mersenne primes, $2^{q}-1, q \in\{2,3,5,7$, $13, \ldots\}$ is at most

$$
\frac{4}{3} \cdot \frac{8}{7} \cdot \frac{32}{31} \cdot \frac{128}{127} \cdots<\frac{4}{3} \cdot \frac{8}{7} \cdot \frac{32}{31} \cdot \frac{64}{63}<\frac{8}{5}
$$


If the prime 7 is missing, this product is less than $7 / 5$; if 3 does not occur, the product is less than $6 / 5$.

The deficiency of $p_{i}^{b_{i}}$ is at most $2^{h_{i}}\left(p_{i}+1\right) / p_{i}^{a_{i}}$, where $h_{i}=\left[\log _{2}\left(b_{i}+1\right)\right]-1$, and is strictly less than this unless $p_{i}$ is a Mersenne prime. So $a_{i}<4$ for each $i$, since otherwise the deficiency of the corresponding factor $p_{i}^{b_{i}}$ is at most $2(3+1) / 3^{4}$, and the product of all deficiencies would be less than $(8 / 81)(6 / 5)<1 / 4$. If $a_{i}=$ 2 or 3 for any $p_{i} \geqslant 5$, the product of the deficiencies would be less than

$$
\max \left\{\frac{2}{5^{2}} \cdot \frac{8}{5}, \frac{8}{7^{2}} \cdot \frac{7}{5}\right\}<\frac{1}{4}
$$

so, with the possible exception of 3 , we may assume that $2^{a+1}-1$ contains no repeated factors.

If $3^{2}\left|2^{a+1}-1,6\right| a+1,3^{2} 7 \mid 2^{a+1}-1$ and then $7 \mid v$, for otherwise the product of the deficiencies is at most $(1 / 7)(7 / 5)<1 / 4$. If $3^{2} \| v$ or $3 \nmid v$, the product is less than $(1 / 9)(6 / 5)<1 / 4$, while if $3^{3}\left|v, 3^{3}\right| 2^{a+1}-1,18\left|a+1,3^{3} 7 \cdot 19 \cdot 73\right| 2^{a+1}-1$ and the product is much less that $1 / 4$. So $3 \| v$, and if $a=5$ we have the driver $2^{5} 3 \cdot 7$. We cannot have a larger, since $2^{a+1}-1$ would contain, in addition to $3^{2} 7$, some factor congruent to $1, \bmod 4$, and the product of the deficiencies would be less than

$$
\frac{4}{9} \cdot \frac{2}{5} \cdot \frac{6}{5}<\frac{1}{4}
$$

We also notice that $2^{a+1}-1$ contains at most one non-Mersenne prime factor, i.e., factor of the form $2^{c} u-1, u$ odd, $u \geqslant 3, c \geqslant 1$, since the deficiency of such a factor is $2^{c} /\left(2^{c} u-1\right)$, which is at most $2 / 5(u=3, c=1)$, or otherwise at most $4 / 11(u=3, c=2)$, and

$$
\frac{2}{5} \cdot \frac{4}{11} \cdot \frac{8}{5}<\frac{1}{4}
$$

It remains to consider $2^{a+1}-1=\left(2^{q_{1}}-1\right)\left(2^{q_{2}}-1\right) \cdots\left(2^{c} u-1\right)$, where $2 \leqslant q_{1}<q_{2}<\cdots$. If $u \geqslant 7$, the product of the deficiencies is less than $(2 / 13)(8 / 5)<1 / 4$, so $u=3$ or 5 . If $c=1, u=3$ (since $2 \cdot 5-1$ is not prime), $2^{c} u-1=5,5\left|2^{a+1}-1,4\right| a+1,15 \mid 2^{a+1}-1$. If $a=3$ we have the drivers $2^{3} 3.5$ and $2^{3} 3$. If $a \geqslant 7$, there is another prime divisor of $2^{a+1}-1$ which is congruent to $1, \bmod 4$, and the product of the deficiencies is at most

$$
\max \left\{\frac{2}{5} \cdot \frac{2}{13} \cdot \frac{8}{5}, \frac{1}{5^{2}} \cdot \frac{8}{5}\right\}<\frac{1}{4}
$$

So we have $c \geqslant 2, q_{1} \geqslant 2, u=3$ or 5 , and, since we have dealt with $1 \leqslant a \leqslant 4$, $a \geqslant 5$. Considerations modulo $2^{\min \left(c, q_{1}\right)+1}$ show that

$$
-1 \equiv\left(2^{q_{1}}-1\right)(-1) \cdots\left(2^{c} u-1\right), \bmod 2^{\min \left(c, q_{1}\right)+1} .
$$

So $\pm 1 \equiv 2^{q} 1+2^{c} u-1$, the choice of sign is minus, the number of Mersenne primes is even (and not zero) and $q_{1}=c$. Now $2^{q_{1}} u \cdot 2^{q_{1}} 2^{q_{2}} \cdots>2^{a+1}$, so 


$$
3+2 q_{1}+q_{2}+\cdots>\log _{2} u+2 q_{1}+q_{2}+\cdots>a+1 \geqslant q_{1} q_{2} \cdots
$$

since $2^{q}-1$ divides $2^{a+1}-1$ just if $q \mid a+1$, and the $q_{i}$ are distinct primes.

This is clearly a contradiction if the number of $q_{i}$ is 4 or more, so there are just two $q_{i}: 3+2 q_{1}+q_{2}>q_{1} q_{2},\left(q_{1}-1\right)\left(q_{2}-2\right)<5, q_{1}=2=c$ and $q_{2}=3$

or 5. Only the latter gives a solution; $u=3$ and $293 \cdot 11 \cdot 31$ is a driver.

The theorem is proved.

Table 1 exhibits some drivers, $d$, and guides, $g$, and the effect on the power of two, $2^{a}$, when $n=d m$ or $g m,(d, m)$ or $(g, m)=1$ and $m=s$, a square or $m=$ ps where $p$ is a prime congruent to $1, \bmod 4$. The odd prime factors of $d$ or $g$ always divide $s(n)$; for a driver $2^{a} \| s(n)$ with the exceptions noted. If $(d, m)>1$, the situation is more complicated.

\section{TABLE 1. Power of 2 dividing $s(n)$}

$\begin{array}{llccc}\text { Driver } \text { or guide } & a & m=s, a \text { square } & m=p s \equiv 1(\bmod 4) \\ d & 2 & 1 & 0 \downarrow & \geqslant 2 \uparrow \\ d & 2 \cdot 3 & 1 & 1 & 1 \\ g & 2^{2} & 2 & 0 \downarrow & 1 \downarrow \\ d & 2^{2} 7 & 2 & 2 & 2 \\ g & 2^{3} & 3 & 0 \downarrow & 1 \downarrow \\ d & 2^{3} 3 & 3 & 2 \downarrow & \geqslant 4 \uparrow \\ g & 2^{3} 5 & 3 & 1 \downarrow & 2 \downarrow \\ d & 2^{3} 3 \cdot 5 & 3 & \geqslant 4 \uparrow & 3 \\ g & 2^{4} & 4 & 0 \downarrow & 1 \downarrow \\ d & 2^{4} 31 & 4 & 4 & 4 \\ g & 2^{5} 3 & 5 & 2 \downarrow & 3 \downarrow \\ g & 2^{5} 7 & 5 & 3 \downarrow & 5 \\ d & 2^{5} 3 \cdot 7 & 5 & \geqslant 6 \uparrow & 6 \\ d & 2^{6} 127 & 6 & 6 & 9 \\ d & 2^{9} 3 \cdot 11 \cdot 31 & 9 & \geqslant 10 \uparrow & \end{array}$

The signs $\downarrow, \uparrow$ indicate that the driver or guide changes "downward" or "upward".

We are examining the statistical and probabilistic evidence concerning boundedness and unboundedness of aliquot sequences in collaboration with M. C. Wunderlich. The probabilistic model is a Markov process. An aliquot sequence is in one of a finite number of states; one of a finite set of drivers and guides is in control, or none of them is. We can calculate the expected "life" of a sequence in one of these states and the expected number of terms in which the driver is retained. "Break probabilities" between pairs of states can also be calculated; for example, that from the $2^{3} 3 \cdot 5$ driver to the 2 driver is zero since direct transition is impossible.

We conclude with a table showing the numbers of sequences starting below $10^{3}$ and below $10^{4}$ which surpass various bounds. The number of distinct sequences at each bound is also given. 
TABLE 2. Numbers of sequences surpassing given bounds

$\begin{array}{lrrrrrrr}\text { bound } & 10^{12} & 10^{15} & 10^{18} & 10^{21} & 10^{24} & 10^{27} & 10^{30} \\ \text { starting below } 10^{3} & 19 & 17 & 16 & 13 & 13 & 13 & 13 \\ \text { distinct } & 10 & 8 & 7 & 6 & 6 & 6 & 6 \\ \text { starting below } 10^{4} & 896 & 820 & 803 & 761 & 751 & ? & ? \\ \text { distinct } & 113 & 106 & 104 & 100 & 98 & ? & ?\end{array}$

We expect to fill in the missing entries in due course, but much computation is needed. As remarked in [5], [6] considerable help from Lehmer's sieve was necessary to push all the sequences beyond $10^{24}$.

Department of Mathematics, Statistics and Computing Science

The University of Calgary

Calgary, Alberta, Canada

Department of Mathematical Sciences

Northern Illinois University

DeKalb, Illinois 60115

1. E. CATALAN, Bull. Soc. Math. France, v. 16, 1887/88, pp. 128-129.

2. HENRI COHEN, “On amicable and sociable numbers," Math. Comp., v. 24, 1970, pp. 423-429. MR 42 \#5887.

3. L. E. DICKSON, "Theorems and tables on the sums of divisors of a number," Quart. $J$. Math., v. 44, 1913, pp. 264-296.

4. RICHARD K. GUY \& J. L. SELFRIDGE, “Interim report on aliquot series," Proc. Manitoba Conf. Numerical Math., Winnipeg, 1971, pp. 557-580.

5. RICHARD K. GUY, D. H. LEHMER, J. L. SELFRIDGE \& M. C. WUNDERLICH, "Second report on aliquot sequences," Proc. 3rd Manitoba Conf. Numerical Math., Winnipeg, 1973, pp. 357-368.

6. RICHARD K. GUY \& J. L. SELFRIDGE, Combined Report on Aliquot Sequences, University of Calgary Math. Research Report No. 225, May 1974.

7. G. AARON PAXSON, "Aliquot sequences (preliminary report)," Amer. Math. Monthly, v. 63, 1956, p. 614; Math. Comp., v. 26, 1972, pp. 807-809.

8. P. POULET, L'Intermédiaire des Math., v. 25, 1918, pp. 100-101. 